\section{Participación ciudadana en un proceso de extensión universitaria orientado a la gestión del riesgo de inundación y anegamiento en Coronel Suárez, Argentina}

\author{
Belén Moretto \\ belen.moretto@uns.edu.ar \\ (iD) orcid.org/0000-0003-4854-8967 \\ Jorge Osvaldo Gentili \\ jogentili@uns.edu.ar \\ (iD) orcid.org/0000-0002-4787-4667
}

Consejo Nacional de Investigaciones

Científicas y Técnicas-Universidad Nacional

del Sur, Argentina
Ambiente y extensión universitaria /

Intervenciones
RECEPCIÓN: 30/04/21

ACEPTACIÓN FINAL: 03/06/21
Citizen participation in a university extension process aimed at flood and waterlogging risk management in Coronel Suárez's district, Argentina

\section{Abstract}

In Coronel Suárez's district, Buenos Aires, Argentina, flood and waterlogging are environmental issues arising from meteorological-climatic events that are likely to be addressed from the university extension. Within the framework of the activities of the university extension project of the Universidad Nacional del Sur called "Inundaciones: ¿qué podemos hacer? Las tecnologías colaborativas en la gestión del riesgo", citizen participation is a key pillar. The activities carried out within the framework of the project aimed at implementing participatory processes to contribute to flood and waterlogging risk management in the Coronel Suárez's district are presented. Working together with society made it possible to reconstruct knowledge about the risk of flood and waterlogging and to reflect on the actions of each actors from the university extension.

Keywords: university extension; participatory workshops; flood and waterlogging risk management; Coronel Suárez..

\section{Participação cidadã em um processo de extensão universitária visando a gestão de risco de inundação e alagamento em Coronel Suárez, Argentina}

\section{Resumo}

No distrito de Coronel Suárez, Buenos Aires, Argentina, as inundações e alagamentos constituem problemas ambientais derivados de eventos de origem meteorológico-climática que provavelmente serão abordados a partir da extensão universitária. No quadro das atividades do projeto de extensão universitária da Universidade Nacional del Sur denominado "Inundaciones: ¿qué podemos hacer? Las tecnologías colaborativas en la gestión del riesgo" ("Inundações: o que podemos fazer? As tecnologias colaborativas na gestão do risco"), a participação cidadã é um pilar fundamental. São apresentadas as atividades realizadas no âmbito do projeto, com o objetivo de implementar processos participativos para contribuir com a gestão de risco de inundações e alagamentos no distrito de Coronel Suárez. O trabalho conjunto com a sociedade permitiu reconstruir o conhecimento sobre o risco de inundações e alagamentos e refletir sobre as ações de cada um dos atores da extensão universitária.

Palavras-chave: extensão universitária; oficinas participativas; gestão de risco de inundação e alagamento; Coronel Suárez. talleres participativos; gestión del riesgo de inundación y anegamiento; Coronel Suárez.

Para citación de este artículo: Moretto, B.; Gentili, J. O. (2021). Participación ciudadana en un proceso de extensión universitaria orientado a la gestión del riesgo de inundación y anegamiento en Coronel Suárez, Argentina. +E: Revista de Extensión Universitaria, 11(14), e0007. doi: 10.14409/ extension.2021.14.Ene-Jun.e0007 


\section{Introducción}

En el suroeste de la provincia de Buenos Aires se erige el Sistema de Ventania, cordón orográfico en forma de arco con rumbo noroeste-sureste (Harrington, 1947) que es la mayor divisoria de aguas provincial y el origen de ríos y arroyos que conforman cuencas exorreicas y endorreicas. En su vertiente norte tiene sus nacientes la cuenca del arroyo Sauce Corto, la cual, luego de atravesar mayormente áreas rurales del partido de Coronel Suárez, vierte sus aguas hacia el centro-oeste de la provincia, en el complejo lagunar Las Encadenadas del Oeste. Ante la ocurrencia de eventos meteorológico-climáticos extremos, la cuenca presenta respuestas hidrológicas diferenciales, lo que da lugar a procesos de inundación y anegamiento que afectan principalmente a las localidades de Coronel Suárez, Huanguelén, Santa Trinidad, San José, Santa María y extensas áreas rurales (Gentili, 2012; Ortuño Cano et al., 2019). Ambos procesos, asociados a excesos hídricos, son consecuencia de factores hidrometeorológicos y climáticos junto a geomorfológicos, topográficos y edafológicos que, en conjunción con infraestructura vial e hidráulica en el área (puentes, terraplenes de rutas y vías férreas, alcantarillas y canales), afectan vastos sectores de la cuenca media y baja. Al respecto, Ortuño Cano et al. (2019) identificaron para el período 1977-2017 un total de 11 eventos de inundación y anegamiento con afectaciones en el sector urbano y rural. A estos se suman los acaecidos en los años 2018, 2019 y 2020, que también produjeron situaciones adversas en la comunidad. Estos eventos constituyen problemáticas ambientales de origen meteorológico-climático que son susceptibles de ser abordadas mediante procesos de extensión universitaria, priorizando la construcción colectiva de conocimiento con el fin de fortalecer los procesos de gestión del riesgo.

El esfuerzo por entender las respuestas de las sociedades ante dichos eventos ha girado tradicionalmente en torno al concepto de riesgo, entendido como el resultado de las interacciones entre peligro (peligrosidad), vulnerabilidad y exposición. Olcina Cantos (2008) define estos tres conceptos: por peligro entiende el fenómeno o proceso de carácter natural que puede originar daños a una comunidad, a sus actividades o al propio ambiente; describe la vulnerabilidad como la pérdida esperable de un determinado bien expuesto y por exposición a la disposición sobre el territorio de un conjunto de bienes a preservar que pueden ser dañados por un peligro natural, en este caso, las inundaciones y anegamientos. El riesgo, como afirma Lavell, "es una construcción social, dinámica y cambiante, diferenciado en términos territoriales y sociales" (2001:4), que involucra a la imágenes cognoscitivas del sujeto concreto, individual o colectivo (Rojas Vilches \& Martínez Reyes, 2011). Por ello, para comprender cómo se construye un riesgo se requiere de la participación de la sociedad y de grupos de interés (Lavell, 2001).

En este sentido, los proyectos de extensión universitaria posibilitan, como plantea Herrera Albrieu (2012), la trasferencia recíproca de conocimientos y saberes entre la universidad y la comunidad, en donde ambas partes se retroalimentan de manera reflexiva e interactiva. La extensión universitaria, entonces, contribuye así al estudio, diseño, monitoreo y evaluación de diferentes problemáticas ambientales (Stein, 2018). Resultan, por tanto, de gran relevancia en los procedimientos participativos, entre los que se encuentran los procesos de gestión del riesgo de desastres debido a que coordinan las relaciones entre ambas partes a partir del trabajo recíproco y contribuyen a la organización y autonomía de los sectores sociales. 
La preparación ante el riesgo de desastre que las sociedades puedan tener implica cierto grado de conocimiento y conciencia respecto de la posibilidad de incidir en el entorno que habitan. En este sentido, Voss \& Wagner (2010) destacan la importancia de impulsar los procesos participativos para evaluar las causas del desastre y aprender de ellos. Participar contribuye a crear condiciones propicias en la práctica y estimula la generación de iniciativas de desarrollo por parte de la población, lo cual refleja las necesidades, aspiraciones y preocupaciones de las partes interesadas. Aspectos que, necesariamente, requieren ser avalados por procesos educativos (Lara San Martín, 2012). Por tanto, la participación ciudadana es un elemento esencial de la gestión de riesgos que posibilita construir una cultura de prevención y aporta al desarrollo sostenible. En este marco de referencia son necesarias actividades que involucren la representación y percepción de la realidad, tomando como punto de partida los excesos hídricos como una problemática de índole ambiental.

En el marco del Proyecto de Extensión Universitaria (PEU) de la Universidad Nacional del Sur (Bahía Blanca) denominado "Inundaciones: ¿qué podemos hacer? Las tecnologías colaborativas en la gestión del riesgo", desde el año 2017 se desarrolla un proceso de extensión universitaria orientado a la gestión del riesgo de inundación y anegamiento en el partido de Coronel Suárez. La participación ciudadana es un pilar fundamental para el desarrollo de un enfoque integral y de relevancia en la reducción y mitigación del riesgo de inundación y anegamiento. En este sentido, las actividades en torno al PEU se encuentran en línea con los objetivos 11 y 13 de los Objetivos de Desarrollo Sostenible (United Nations General Assembly, 2015) en pos de construir sociedades cada vez más resilientes y sostenibles.

El proyecto está conformado por un grupo multidisciplinario de docentes, alumnos y no docentes de los departamentos de Geografía y Turismo y de Ciencias e Ingeniería de la Computación que trabajan en conjunto con actores e instituciones de la localidad de Coronel Suárez (Gentili et al., 2018). En este marco de referencia, siguiendo a Fernández (2021), se entiende la extensión como un proceso crítico y reflexivo donde la universidad se proyecta a la comunidad a partir de un trabajo conjunto y de intercambio de saberes, al mismo tiempo que busca generar profesionales comprometidos con la transformación social.

Para el área de estudio, miembros del PEU realizaron trabajos vinculados a problemáticas ambientales (específicamente, inundaciones y anegamientos) abordadas desde la extensión universitaria: Gentili et al. (2016); Gentili et al. (2018); Moretto et al. (2019) y Moretto et al. (2020). En este sentido y continuando la línea de los trabajos mencionados, se avanzó desde la extensión con comunidades educativas formales de la localidad de Coronel Suárez a fin de fomentar una cultura de prevención y atención de desastres y de participación social en procesos de gestión del riesgo de inundación y anegamiento. Por lo expuesto con anterioridad, el objetivo de este trabajo es presentar las actividades llevadas a cabo en el marco del PEU destinadas a implementar procesos participativos para contribuir a la gestión del riesgo de inundación y anegamiento en el partido de Coronel Suárez.

La sensibilización y la formación en torno a los conceptos sobre el riesgo y su gestión se convierten en una de las actividades elementales para la reducción de desastres y son las comunidades educativas los verdaderos actores de la gestión prospectiva del mismo. De este modo, el presente trabajo está relacionado con la elaboración y aplicación de medidas no estructurales que posibiliten construir representaciones para la toma de deci- 
siones, elaborar programas y proyectos integrales, aumentar el conocimiento colectivo de la ciudadanía, disminuir las vulnerabilidades y por lo tanto el riesgo ante la ocurrencia de inundaciones y anegamientos.

El artículo se estructura en tres apartados. Primeramente, se exponen de manera descriptiva las etapas y acciones llevadas a cabo para la construcción de los talleres participativos en el marco del proyecto y las características generales para implementarlos. Luego se presentan los principales resultados de los talleres bajo el enfoque de una metodología cualitativa dispuestos en dos fases: la primera de diagnóstico y sensibilización y la segunda de ellas de reconstrucción colectiva de la percepción del riesgo. Por último, se exhiben las reflexiones finales del trabajo en conjunto con instituciones educativas, la importancia de la participación ciudadana en la gestión del riesgo de inundación y anegamiento y el impacto de la experiencia para el grupo de trabajo y para sus futuras líneas de acción.

\section{Construcción de talleres participativos desde la extensión universitaria}

Del marco teórico-metodológico considerado para la gestión del riesgo (Lavell, 2001; Cardona, 2001; Olcina Cantos, 2008; Natenzon \& Ríos, 2015; Challies et al., 2016; United Nations General Assembly \& United Nations Office for Disaster Risk Redution, 2016) se desprende que es en lo local en donde se construyen y materializan los riesgos y que es fundamental la participación ciudadana para desarrollar una comunidad más resiliente; por tanto es allí y junto a ellos donde debe iniciarse el proceso para conocer, reducir y manejar esos riesgos.

Se programaron talleres de carácter participativo con actividades específicas para favorecer el aprendizaje significativo y el intercambio de experiencias. Para el conjunto de actividades realizadas en los mismos, la metodología utilizada fue cualitativa del tipo investigación-acción (Hernández Sampieri et al., 2014) y se planteó la incorporación de acciones concretas con la participación de distintos grupos en el sector educativo formal (Alpízar, 2009). Se hizo un trabajo conjunto, durante el transcurso del año 2019, con dos instituciones educativas de la localidad de Coronel Suárez: la Escuela de Educación Secundaria (EES) № 6 Paraje El Relincho y el Instituto Superior de Formación Docente (ISFD) № 160. En el marco de la extensión, los talleres no son solo eventos formales educativos sino que el compromiso se centra en aprender a aprender y complementar el saber técnico-académico con el saber y experiencia local para que se puedan reducir los riesgos de desastres y responder adecuadamente a las emergencias (Ferradas et al., 2006).

Las actividades en esta etapa del proceso de extensión universitaria (iniciado con el PEU mencionado) se planificaron en dos fases, en concordancia con la gestión del riesgo de desastres. La primera etapa consistió en el conocimiento y sensibilización acerca de la problemática de las inundaciones y anegamientos en el territorio y de los conceptos relacionados con el riesgo, sus factores y gestión (Figura 1). Así, se realizaron actividades que contribuyeran a comprender el conocimiento de la comunidad respecto del riesgo y las problemáticas vinculadas a los excesos hídricos. Se elaboró una encuesta a ejecutarse por muestreo aleatorio simple (Hernández Sampieri et al., 2014) con criterios relativos a la naturaleza de la problemática y su gestión destinadas a estudiantes y docentes de las instituciones (Figura 1). La segunda etapa consistió en la aplicación de técnicas dentro del enfoque metodológico de Investigación-Acción Participativa sugerido por Orlando Falls Borda (Ortiz \& Borjas, 2008). 
Se hicieron dos talleres destinados a la elaboración de cartografía social para explorar la percepción del riesgo de inundación y anegamiento de la comunidad educativa (Figura 1). Estos mapeos forman parte de las "nuevas prácticas cartográficas" concebidas como actividades colectivas y horizontales que surgen de un proceso de intercambio y de debate en el que los actores sociales son sujetos claves para el entendimiento de las realidades territoriales (Castro Jaramillo, 2016; Martin Silva et al., 2019). La cartografía social es una construcción colectiva porque representa virtudes, lazos reales en donde el trabajo en conjunto implica una tarea compartida, con fuerte intercambio de ideas, debate sobre acciones, objetos, y conflictos, y finalmente un consenso (Diez Tetamanti, 2012). Para la preparación del taller se consultaron diferentes fuentes (Castro Jaramillo, 2016; Chambers, 2006; Diez Tetamanti, 2012; Lafuente \& Hornillo, 2017; UNGRD- IEMP, 2017), en tanto que los elementos que se tuvieron en cuenta para llevar a cabo el mismo se presentan en la Tabla 1.

Tabla 1. Consideraciones generales para el desarrollo de los talleres

\begin{tabular}{|c|c|}
\hline Consideraciones distintivas & Descripción \\
\hline Marco de referencia & $\begin{array}{l}\text { En el marco de la Gestión del Riesgo de Desastre, esta técnica se puede desa- } \\
\text { rrollar ya sea por medio de la construcción de mapas o dibujos elaborados por } \\
\text { la misma comunidad de forma grupal o por medio de mapas construidos ya sea } \\
\text { por un experto o una institución. Aquí se optó por esta última modalidad a los } \\
\text { fines prácticos del objetivo del taller. }\end{array}$ \\
\hline Actores sociales & Estudiantes, profesores y directivos del nivel secundario y superior. \\
\hline Espacio físico & Los establecimientos de la EES N ${ }^{\circ} 6$ y del ISFD N ${ }^{\circ} 160$. \\
\hline Materiales para trabajar & $\begin{array}{l}\text { Mapas base de las localidades y el partido de Coronel Suárez, lapiceras de } \\
\text { colores y cuadernos. }\end{array}$ \\
\hline
\end{tabular}

Fuente: elaboración propia, 2021

Con esta dinámica, entonces, se encuentran procesos de representación y percepción del riesgo de inundación y de anegamiento. Como primera instancia, se propone realizar una explicación de los principales resultados obtenidos en los talleres participativos y cómo dichas experiencias ayudan a entender de mejor manera los eventos de excesos hídricos en el partido de Coronel Suárez y la relación de los estudiantes (como primer eslabón dentro de los procesos de gestión del riesgo de inundación y anegamiento) con el territorio (Figura 1).

\section{Participación ciudadana en los procesos de gestión del riesgo de inundación y anegamiento desde los PEU}

Una aproximación inicial hacia la participación ciudadana se lleva a cabo a partir de la inclusión de la comunidad educativa de la localidad de Coronel Suárez en la gestión del riesgo de inundación y anegamiento. Se trabajó en forma conjunta mediante la modalidad taller como un dispositivo con grupos que posibilita efectuar una serie de actividades en torno a dos fases de aplicación: una de diagnóstico y sensibilización y otra de reconstrucción colectiva de la percepción del riesgo. Se presentan a continuación los principales resultados y aportes obtenidos de ambas etapas. 
Figura 1. Enfoque teórico - metodológico

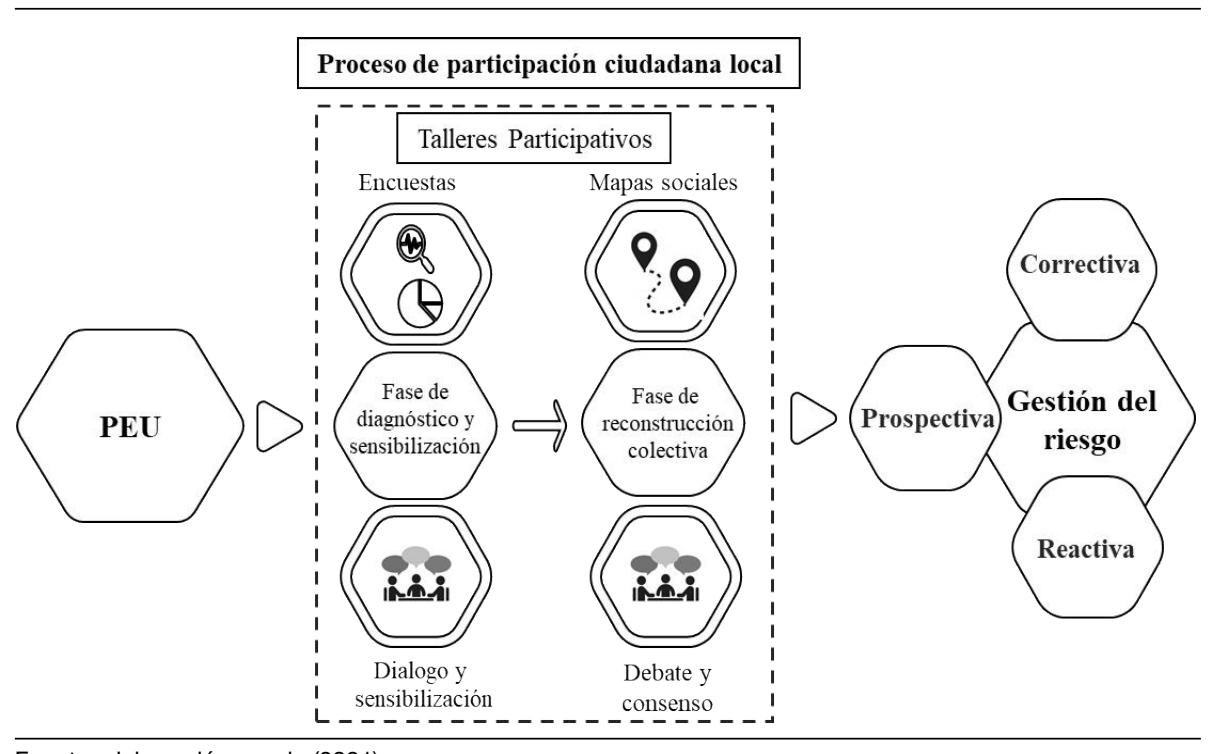

Fuente: elaboración propia (2021)

\section{Fase de diagnóstico y sensibilización: aproximaciones y diálogos en torno al riesgo de inundación y anegamiento}

En primera instancia, se realizó un total de 77 encuestas. Los resultados fueron muy valiosos, ya que expusieron las dificultades que afronta la comunidad educativa con relación a los eventos de origen hidrometeorológico. De acuerdo con la información obtenida, la población no es indiferente a la ocurrencia de los mismos, sin embargo, la percepción del riesgo de inundación es baja. Cerca de la mitad de los encuestados no pudo establecer una clara definición de ambos eventos y consideró el anegamiento como consecuencia de la inundación, ya que las diferencias se simplifican a las afectaciones estructurales que podría ocasionar uno u otro.

Las encuestas revelaron que no existe un nivel alto de conciencia sobre lo que conlleva la ocurrencia de los eventos de excesos hídricos en el partido de Coronel Suarez. Muchas de las personas, a la hora de responder al interrogante en cuanto a su experiencia ante la ocurrencia de este tipo de eventos, recuerdan la inundación de 1985 y 1992 (ambas de gran envergadura espacial, social y económica para la región) y una proporción menor nombra los eventos de 2019 (se corresponde con las edades de entre 18 y 19 años), como también los acaecidos en 2014 y 2016. Asimismo, un $21 \%$ detalla no haber vivenciado tales eventos y el resto de las personas reconoció haber vivido eventos de inundación pero no recuerda los años. Con relación a ello, es importante destacar dos aspectos: uno es que la población encuestada suele tener presente los eventos de mayor impacto en su territorio y/o aquellos ocurridos más recientemente. Otro es que los individuos de rangos etarios más jóvenes solo referencian eventos recientes en el tiempo, lo cual conduce a reflexionar en torno a la carencia de procesos de sensibilización. Ambos aspectos dan cuenta de la falta de memoria individual, que deriva en colectiva, ante la ocurrencia de inundaciones y anegamientos que impactan en su entorno, lo cual contribuye a que la sociedad sea más vulnerable. Estos 
resultados podrían atribuirse a una carencia de información respecto de los impactos que generaron los eventos del pasado y al desconocimiento del grado de responsabilidad de la comunidad en los procesos de gestión del riesgo de inundación y anegamiento, lo que ocasiona que no se construya, posiblemente, una percepción de riesgo ante ellos. De este análisis se desprende la prevalencia aún de un enfoque de la gestión desde la emergencia.

Conforme a la información obtenida, afirman que las causas devienen en naturales debido a la ocurrencia de lluvias intensas, crecida de los arroyos que provienen de las sierras y la morfología del terreno. Entre las respuestas se indica con gran frecuencia la necesidad de obras o infraestructuras públicas, y entre ellas destacan la deficiencia de redes de alcantarillado, de desagües y bocas de tormenta. Se menciona también la contaminación por parte de la población particularmente el desecho de basura en áreas indebidas, y como consecuencia de ello las bocas de tormenta y los desagües tapados y canales sin mantenimiento ni limpieza. En menor medida, señalan la falta de planificación urbana, cambios en el curso natural del arroyo, falta de conocimiento, de prevención y de interés. Al respecto, de las causas que se identificaron, Olleros (2020) plantea una serie de mitos socialmente extendidos. En cuanto a la falta de limpieza de los cauces que se menciona entre las causas, indica que es un erróneo eliminar sedimentos y vegetación del sistema fluvial ya que son las crecidas las que limpian y renuevan los cauces. De igual manera, otro punto vinculado a la necesidad de obras, específicamente, de ampliar la sección de desagüe, es igualmente erróneo puesto que se asienta en la idea de que toda acumulación natural de sedimentos es negativa debido a que tapona o hace crecer al cauce incrementando los desbordamientos. El autor agrega que una crecida reconstruye el cauce, dota al sistema de resiliencia y que, ante cualquier acción humana, esa autorregulación natural se altera.

Otro de los puntos que se consultaron fueron las afectaciones ante la ocurrencia de los eventos de excesos hídricos. Identificaron las calles como los elementos más afectados, seguidos por las redes de alcantarillado, viviendas y la caída de árboles. Durante el análisis de las encuestas se hizo contundente el hecho de que hace falta mayor información y sensibilización respecto de los eventos de inundación y anegamiento, sus causas y posibles consecuencias, debido a que el desconocimiento conlleva a un aumento de la vulnerabilidad en el territorio. Se descubrió que los participantes otorgan mayor valoración al apoyo social prestado por los Bomberos Voluntarios y es necesario recalcar que todas las personas involucradas resaltaron su accionar sobre el territorio con referencia a las atribuciones que les fueron otorgadas a los demás actores públicos. En cuanto a la pregunta acerca de si consideran que tienen las capacidades necesarias para contribuir a reducir y mitigar los efectos de estos peligros, cerca de la mitad de los individuos encuestados se percibe como capaz de dar soluciones, brindar información con charlas de prevención, contribuir con la limpieza de espacios públicos y evitar tapar bocas de desagüe. Algunos de ellos mencionaron que utilizaban la aplicación InundApp (presentada por el equipo en talleres anteriores) como herramienta para contribuir a reducir el riesgo de inundación y anegamiento. No obstante, más de la mitad de las personas encuestadas se reconoce como incapaz de contribuir y menciona como las causas de ello que no es posible ayudar desde su rol y la falta de conocimiento e instrucción en la temática debido a que, según sus propias palabras, "no se puede hacer", o no conoce la forma de ayudar o considera que son cuestiones que corresponden a decisiones políticas o para la gente especialista en el área. 
Las encuestas posibilitaron evaluar cuál es la situación inicial en la que se encontraba la comunidad educativa en el año 2019 respecto del riesgo de inundación y anegamiento y su gestión en el partido de Coronel Suárez. Estos resultados se asimilan con los obtenidos en una encuesta realizada a los habitantes de áreas urbanas del partido en el año 2020, para la cual se obtuvo una muestra de 150 personas.

En segunda instancia, se desarrollaron talleres de sensibilización en ambas instituciones, en las cuales se debatieron los conceptos en torno al riesgo como una construcción social mediante diferentes estrategias y actividades. En la EES $N^{\circ} 6$ se plantearon preguntas para debatir de manera grupal y partir de los conocimientos previos de los estudiantes: ¿Qué son las inundaciones y anegamientos? ¿Alguna vez sufrieron una inundación o anegamiento? ¿Cómo los afectó? ¿Cuáles fueron las medidas o acciones que llevaron a cabo con su familia? Se compartieron experiencias en torno a inundaciones pasadas y a cómo debieron afrontarlas. Complementariamente, se utilizaron técnicas audiovisuales como apoyo para comprender la relación entre precipitaciones e inundaciones y el análisis de medidas preventivas. Se recurrió, a su vez, a actividades de lectura para la comprensión y reflexión en torno a los conceptos asociados al riesgo, ya que desconocían lo que significaba cada uno de ellos. Este taller posibilitó compartir conocimientos entre ambas partes y al mismo tiempo obtener un diagnóstico preliminar del grado de implicancia en la temática para continuar trabajando en virtud de lo observado.

En la misma línea, en el ISFD N ${ }^{\circ} 160$ se abordó la problemática de las inundaciones y anegamientos. Para ello se analizaron en forma conjunta dos recursos didácticos para trabajar en el espacio áulico: la prensa escrita y las nuevas tecnologías. Se compartieron ideas para su aplicación futura y se solicitó elaborar una propuesta de estructuración didáctica con la temática de las inundaciones y anegamientos en el partido de Coronel Suárez mediante la utilización de algunos de los recursos didácticos presentados. Estas actividades permiten que los estudiantes cuenten con herramientas para su futuro desempeño como docentes y adentrarlos en la temática de los eventos de excesos hídricos como problemáticas ambientales desde la escala local.

Lo observado de la aplicación de las encuestas y el desarrollo de los talleres en esta fase denotan tres problemas: el deficiente conocimiento que posee la población en relación con la hidrografía y factores asociados al relieve de su partido; el escaso entendimiento de la problemática de las inundaciones y anegamientos; y la falta de memoria colectiva en torno a los impactos que ocasionan. Estas deficiencias configuran escenarios de riesgo que es necesario reducir.

Las actividades ejecutadas en esta fase se realizaron con la finalidad de que los participantes se identificaran como parte de la solución a los problemas planteados y tomaran un rol proactivo en la transformación y mejoramiento de su territorio. Por tanto, se considera, al igual que Miceli et al. (2008), que la sensibilización de la comunidad educativa sobre los riesgos de desastres puede influir en sus actitudes para ser más proactiva, y es por ello que la concientización y la educación pueden empoderar a la población para que participe en los procesos de reducción del riesgo (Federación Internacional de Sociedades de la Cruz Roja y Media Luna Roja, 2011). 


\section{Fase de reconstrucción colectiva de la percepción del riesgo: las inundaciones en la cuenca del arroyo Sauce Corto}

En consonancia con lo anterior, se desarrolló el taller de acciones colectivas en torno al riesgo de inundación y anegamiento mediante la elaboración, como experiencia inicial, de una cartografía social. Inicialmente se realizó una introducción en línea con la dinámica preestablecida con el fin de dar conocer a los participantes la propuesta de trabajo, es decir, lo que implicaba la cartografía social, el objetivo del taller y los pasos metodológicos a seguir. Es importante tener en cuenta que la metodología fue conversada con los directivos y profesores de ambas instituciones educativas en una etapa previa a su aplicación.

Una vez establecidas las expectativas de logro y el funcionamiento de la herramienta, los estudiantes y profesores involucrados se organizaron en grupos. Se entregaron a cada uno de los grupos mapas base del partido de Coronel Suarez y de las principales localidades del partido (Coronel Suárez, Huanguelén y las tres colonias: Santa Trinidad, San José y Santa María) elaborados por el equipo del PEU. Cada grupo contaba con una persona que debía anotar todo el proceso de manera reflexiva focalizando en las dificultades a resolver y aspectos a resaltar a la hora de hacer el taller. Como primer paso, los estudiantes debieron marcar con color celeste la hidrografía del partido. Si bien algunos grupos pudieron desarrollarlo con claridad, la información recabada fue deficiente. Muchos de ellos expresaron su dificultad para reconocer y trazar los arroyos. En particular, en uno de los grupos en el ISFD N ${ }^{\circ} 160$ manifestó que había surgido confusión con los nombres de los arroyos y ríos cercanos al Sistema de Ventania (más alejados de los centros urbanos), lo cual evidenció la necesidad de contar con talleres cuyos contenidos versen sobre la hidrografía y aspectos relativos al relieve de su territorio. Durante este proceso se pidió a los grupos del ISFD $N^{\circ} 160$ que señalaran en el mapa aquellas estancias, parajes y estaciones icónicas para ellos. Uno solo de los grupos pudo localizarlos, sin problemas, a partir del conocimiento y el debate entre las partes integrantes, no solo las estancias sino también obras hidráulicas como puentes y el Partidor Piñeyro, infraestructura hidráulica de gran relevancia para la región.

Como segunda instancia, en ambas instituciones, trazaron con color rosa áreas que ellos consideraron que se inundaban o anegaban frecuentemente. Se debatieron y recordaron situaciones particulares, como las inundaciones de 2014, 2016 y 2017 que afectaron no solo a productores que no pudieron levantar sus cosechas, sino también a las actividades socioeconómicas en general. Cada individuo aportó información relativa a áreas que recordaba según su experiencia para luego plasmarla en el mapa. Muchos de los participantes, particularmente los de la EEES $N^{\circ} 6$, compartieron experiencias vividas con sus familias en relación con eventos de inundación en sus campos y que, como en muchas ocasiones, los dejaba varados durante días sin poder asistir a clases. Al mencionar estas experiencias, se les pidió que intentaran ubicar dichas estancias en el plano. Los resultados fueron no concluyentes debido a que se les dificultó localizarlas espacialmente.

Algunos de los productos cartográficos se visualizan en la Figura 2. Las áreas que consideraron propensas a anegarse e inundarse se encuentran en inmediaciones a las localidades de Cura Malal, Cascada, Huanguelén, sectores de la localidad de Coronel Suárez y cercanías del área serrana de Coronel Suárez (suroeste del municipio). La mayoría de los grupos identificó sectores próximos a las colonias de San José, Santa María, áreas del Balneario 
Municipal Samuel Davies, ubicado a $15 \mathrm{~km}$ de la localidad de Coronel Suárez, a la vera del arroyo Sauce Corto, así como áreas rurales cercanas al Partidor Piñeyro. Cabe destacar el énfasis que se otorgó a estas últimas áreas durante el desarrollo del taller.

Asimismo, a los estudiantes del ISFD № 160 se les facilitó una hoja con los planos de las localidades de Coronel Suárez, Huanguelén, Santa Trinidad, San José y Santa María, para que plasmaran las áreas que consideraban propensas a la ocurrencia de eventos de excesos hídricos. Se destaca que los grupos señalaron los barrios nuevos creados con planes municipales y federales como los de mayor riesgo (Figura 2).

Figura 2. Productos cartográficos realizados mediante participación comunitaria educativa en la EES $\mathrm{N}^{\circ} 6$ y en el ISFD $\mathbf{N}^{\circ} 160$
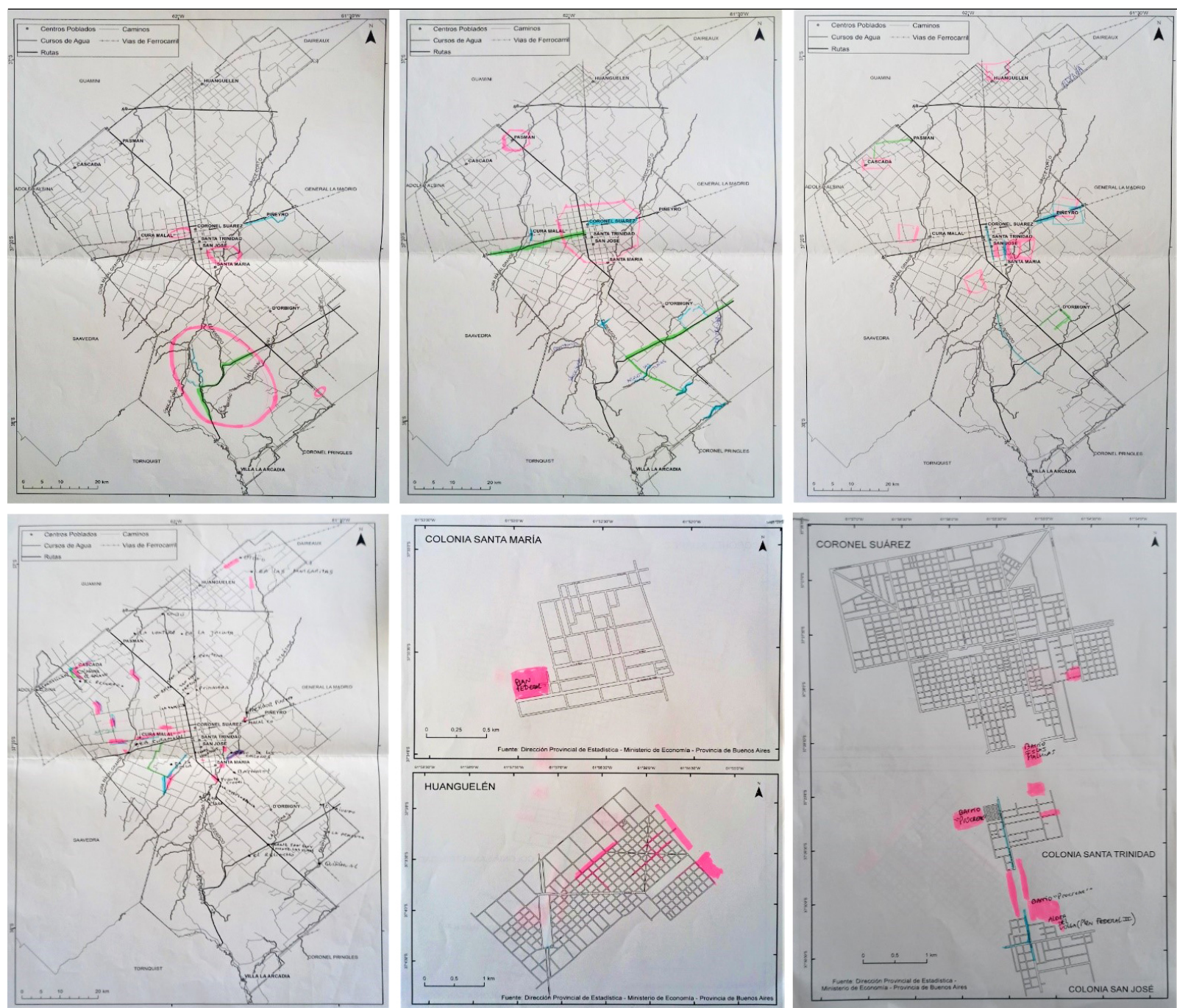

Fuente: elaboración de los autores (2021).

La siguientes dos etapas consistieron en marcar con líneas azules los caminos que se inundan o anegan y con líneas verdes las vías de escape que conocían del municipio (Figura 2). 
Con relación a los caminos o rutas que se anegan o inundan, los participantes coincidieron en el Camino La Emilia y el G 8 (ambos localizados en el suroeste de la localidad de San José hacia el sector oeste de la Ruta Provincial $N^{\circ} 85$ ). Al respecto, dos familias residentes del área rural del partido, específicamente en el cuartel VII, afirmaron que en dichos caminos el agua permanecía 48 horas aproximadamente y suelen estar anegados hasta por 10 meses, por lo cual expresaron: "al vivir en el campo es imposible salir ante una urgencia, llevar los chicos al colegio, se complica mucho". Como consecuencia directa, mencionaron: "destrucción parcial y/o total de caminos rurales; cuantiosas pérdidas económicas y productivas por anegamientos de los lotes y pérdidas de fertilidad por lixiviación". En cuanto a las vías de escape, a todos los grupos se les dificultó identificarlas. En cuanto a ello, indicaron que ante la ocurrencia de una inundación utilizaban caminos vecinales, lindantes a los caminos que suelen anegarse (Figura 2).

La actividad cerró con la presentación de resultados, donde un representante de cada uno de los grupos relataba la experiencia del taller, los pasos metodológicos y las dificultades que habían tenido que afrontar. Las principales dificultades se relacionan con la localización de ríos y arroyos y sus respectivos nombres. Muchos de ellos fueron localizados y nombrados en áreas donde no corresponde. Del mismo modo, tuvieron problemas para ubicarse espacialmente en primera instancia, sumados al desconocimiento de la localización de algunas estancias y campos que les fueran de referencia. Finalmente, se realizó una breve discusión sobre el carácter crítico y reflexivo de la actividad. A partir del relato del otro y del mapa como soporte, pudieron recordar sobre eventos de inundación y experiencias en torno a ello, por lo que podemos corroborar el carácter de ayuda memoria de este tipo de técnicas.

La experiencia fue muy significativa en ambas instituciones. Posibilitó, por un lado, hacer visible el peligro frente a eventos de inundación y anegamiento y, por otro lado, la construcción colectiva de escenarios de riesgos debido a que habitan y transitan en el territorio, promoviendo la reconstrucción de la memoria colectiva (Altez, 2016) de la sociedad. Una memoria colectiva que apunta a la consolidación simbólica de la sociedad, que incorpora aquellos aspectos que le recuerdan la existencia de los peligros con los cuales convive. Es preciso modificar el abordaje del problema, y en este sentido un aspecto clave en los procesos de gestión del riesgo de desastres es la educación de la población, la conservación de la memoria y la experiencia y la sensibilización en el conocimiento hidrológico (Ollero Ojeda, 2020). Reconstruir la memoria permite que la problemática esté latente con el fin de hallar herramientas para mitigar y gestionar el riesgo.

\section{Valoración y reflexiones finales}

La vigencia de la problemática ambiental abordada remite al hecho de que es un tema de interés dados los impactos que conlleva su ocurrencia en el territorio. Comprender la relación sociedad-naturaleza en cualquier lugar es factible gracias a la información objetiva de expertos e instituciones que estudian los procesos que allí acaecen complementados con el saber subjetivo de la comunidad local.

La participación ciudadana, enmarcada en los procesos de extensión universitaria, es relevante a la hora de mitigar o gestionar los riesgos. Esto se debe a que la incorporación y el trabajo mancomunado con instituciones locales fortalecen los mecanismos de gestión de 
riesgo. En esta línea, el desarrollo de proyectos de extensión universitaria posibilita interrelacionar el saber académico con el saber local y con las experiencias que conforman el territorio y, por tanto, con los escenarios de construcción del riesgo.

La realización de talleres a través del PEU hizo posible que la sociedad se involucrara y fuera partícipe de los procesos de gestión del riesgo de desastres. El trabajo con los establecimientos de educación del partido de Coronel Suárez permitió comprobar el estado actual referido a la información y percepción que poseían con relación al riesgo de inundación. La fase de diagnóstico y sensibilización mediante dicha modalidad supone no solo un intercambio de ideas y conceptos de ambas partes sino también que los actores intervinientes se apropien, internalicen y se reconozcan como un eslabón de gran relevancia en los procesos de gestión. La segunda fase, relativa a los talleres de cartografía social, visibilizó el carácter reflexivo, crítico y productivo de la herramienta. Los mapas construidos son el primer paso para realizar réplicas de dicha herramienta en todo el partido de Coronel Suárez. Su aplicación favoreció la reconstrucción de la memoria colectiva debido al debate y a que se compartieron experiencias y vivencias en torno a la problemática. Sus relatos y vivencias nutrieron al equipo del PEU y posibilitaron visibilizar las líneas en las cuales es factible continuar trabajando. Este recurso y su futura complementación con información proveniente de organismos públicos y de resultados académicos pueden mejorar el conocimiento existente sobre el riesgo de inundaciones y ayudar a desarrollar la resiliencia de la comunidad en su conjunto.

La aplicación de las distintas herramientas en los talleres puso de manifiesto no solo la falta de memoria colectiva sino también la ausencia de una cultura de prevención y el desconocimiento del grado de participación y responsabilidad en la gestión de la problemática. Estos resultados evidencian la prevalencia aún de un enfoque de la gestión desde la emergencia. Hace falta una acción educativa y de divulgación que cumpla con criterios generalizables. Se deben elaborar más talleres para compartir información relativa a la dinámica hidrográfica del partido de Coronel Suárez. Conocer la dinámica sociedad-naturaleza posibilita reconocer al arroyo como un recurso pero a su vez como un peligro. Es por ello que es preciso desarrollar más actividades de campo para el mantenimiento de la relación establecida a través de las acciones del PEU que impulsen una cultura de prevención.

El trabajo con ambas instituciones locales educativas potenció el acervo experiencial y académico de cada uno de los integrantes y del grupo de trabajo del PEU. No solo permitió la construcción y transferencia de conocimientos asociados al riesgo de inundación sino que también dio lugar a la reflexión respecto de las propias prácticas que se realizan en el territorio. En este sentido, los resultados de las actividades de cada taller dan cuenta del impacto positivo de las acciones llevadas a cabo y permiten delinear futuras líneas de investigación como también repensar mecanismos de acción tendientes a fomentar una cultura de prevención. El proyecto se encuentra en constante movimiento y transformación en cuanto a las necesidades de la población y a las propias del grupo de trabajo.

\section{Referencias bibliográficas}

Alpízar, M. (2009). Educación y reducción de riesgos y desastres en Centroamérica: gestión del riesgo. En Educación y reducción de riesgos y desastres en Centroamerica: Gestión del riesgo. Coordinación Educativa y Cultural Centroamericana (CECC/SICA). 
Altez, R. (2016). Nacionalización de las memorias colectivas y reproducción de riesgos en regiones fronterizas latinoamericanas. Anuario de Estudios Americanos, 73(1), 319-350. https://doi.org/10.3989/aeamer.2016.1.11 Cardona, O. D. (2001). La necesidad de repensar de manera holística los conceptos de vulnerabilidad y riesgo: una crítica y una revisión necesaria para la gestión. International Work-Conference of Vulnerability in Disaster Theory and Practice.

Castro Jaramillo, M. L. (2016). Cartografía Social como recurso metodológico en los procesos de planeación participativa de un territorio incluyente. El caso del Plan Parcial de Renovación Urbana "El Triángulo de Fenicia" en la ciudad de Bogotá. Pontificia Universidad Javeriana, Bogotá. www.iranesrd.com

Challies, E.; Newig, J.; Thaler, T.; Kochskämper, E. y Levin-Keitel, M. (2016). Participatory and collaborative governance for sustainable flood risk management: An emerging research agenda. Environmental Science and Policy, 55, 275-280. https://doi.org/10.1016/j.envsci.2015.09.012

Chambers, R. (2006). Participatory mapping and Geographic Information Systems: Whose map? Who is empowered and who disempowered? Who gains and who loses? The Electronic Journal of Information Systems in Developing Countries, 25(1), 1-11. https://doi.org/10.1002/j.1681-4835.2006.tb00163.x

Diez Tetamanti, J. M. (2012). Cartografía social. Herramienta de intervención e investigación social compleja. El vertebramiento inercial como proceso mapeado. En Diez Tetamanti (Comp). \& Escudero, B. (Ed.). Investigación e intervención desde las ciencias sociales, métodos y experiencias de aplicación (pp. 13-24). Universidad de la Patagonia.

Federación Internacional de Sociedades de la Cruz Roja y de la Media Luna Roja (IFRC) (2011). Guía: Para la sensibilización y educación pública sobre la reducción de riesgos de desastres. www.ifrc.org

Fernández, P. (2021). La universidad y su vinculación con el medio social: políticas de extensión en la Universidad Nacional del Sur (1986-2019). En Universidades comprometidas con el futuro de América Latina. IV Congreso de Extensión Universitaria de AUGM (pp. 150-162). Asociación de Universidades Grupo Montevideo (AUGM).

Ferradas, P.; Vargas, A. y Santillán, G. (2006). Metodologías y herramientas para la capacitación en gestión de riesgo de desastres. Journal of Chemical Information and Modeling, 53(9). Instituto Nacional de Defensa Civil (INDECI), Soluciones Prácticas (ITDG).

Gentili, J. O. (2012). Hidrografía del Arroyo Sauce Corto aplicada al estudio de las inundaciones y anegamientos. Departamento de Geografía y Turismo. Universidad Nacional del Sur.

Gentili, J. O.; Moretto, B.; Martínez, D.; Gzain, O.; Marziali, L. y Campo, A. M. (2016). Requerimientos de una aplicación para dispositivos móviles destinada a la gestión del riesgo de inundación en la cuenca del arroyo Sauce Corto. 3ras. Jornadas de Tecnologías de Información Geográfica Del Sur Argentino.

Gentili, J. O.; Fernández, M. E.; Zapperi, P. A. y Silva, A. M. (2018). Tecnología y extensión universitaria: los Sistemas de Alerta Temprana Colaborativos en la gestión del riesgo de inundación. +E: Revista de Extensión Universitaria, 8(8), 161-173. https://doi.org/10.14409/extension.v8i8.ene-jun.7725

Harrington, H. (1947). Explicación de las Hojas Geológicas 33m (Sierra de Curamalal) y 34 m (Sierra La Ventana). Provincia de Buenos Aires. Boletín de La Dirección de Minería y Geología, 61, 1-43.

Hernández Sampieri, R.; Fernández Collado, C. y Baptista Lucio, P. (2014). Metodología de la Investigación. McGraw-Hill.

Herrera Albrieu, M. L. (2012). Una mirada sobre la extensión universitaria en Argentina. http://www.bdigital. unal.edu.co/7472/

Lafuente, A. y Hornillo, P. (2017). Cómo hacer un mapeo colectivo. La aventura de aprender.

Lara San Martín, A. (2012). Percepción Social en la Gestión del Riesgo de Inundación en un área Mediterránea (Costa Brava, España). Universidad de Girona. http://hdl.handle.net/10803/98249 
Lavell, A. (2001). Sobre la gestión del riesgo: apuntes hacia una definición. Scripta Nova-Revista. http:// cidbimena.desastres.hn/pdf/spa/doc15036/doc15036-contenido.pdf

Martin Silva, V. B.; Zabala, M. E. y Fabra, M. (2019). Cartografía Social como recurso metodológico para el análisis patrimonial. Experiencias de mapeo en Miramar (Córdoba, Argentina). Perspectiva Geográfica, 24(2), 125-148. https://doi.org/10.19053/01233769.8631

Miceli, R.; Sotgiu, I. y Settanni, M. (2008). Disaster preparedness and perception of flood risk: a study in an alpine valley in Italy. Journal of Environmental Psychology, 28(2), 164-173. https://doi.org/https://doi. org/10.1016/j.jenvp.2007.10.006

Moretto, B.; González, M.; Ortuño Cano, M.; Silva, A. y Gentili, J. (2019). Tecnologías colaborativas y participación comunitaria: análisis preliminar de resultados de InundApp. VII Congreso Nacional de Geografía de Universidades Públicas y XXI Jornadas de Geografía de La Universidad Nacional de La Plata.

Moretto, B.; Ortuño Cano, M. y Gentili, J. (2020). Experiencias de participación ciudadana en la gestión del riesgo de inundación (Coronel Suárez). $4^{\circ}$ Jornadas Nacionales y Las $1^{\circ}$ Jornadas Provinciales de Agua y Educación: Desafíos En Un Mundo En Continua Transformación.

Natenzon, C.; Ríos, D. (2015). Riesgos, catástrofes y vulnerabilidades : aportes desde la geografía y otras ciencias sociales para casos argentinos. Imago Mundi.

Olcina Cantos, J. (2008). Cambios en la consideración territorial, conceptual y de método de los riesgos naturales. Scripta Nova: Revista Electrónica de Geografía y Ciencias Sociales, 12. https://www.raco.cat/index.php/ ScriptaNova/article/view/114794

Ollero Ojeda, A. (2020). Crecidas, inundaciones y resiliencia: restauración fluvial contra los falso mitos. En López Ortiz, M. I. y Melgarejo, J. (Eds.), Riesgo de inundación en España: análisis y soluciones para la generación de territorios resilientes (pp. 549-567). Universitat d'Alacant.

Ortiz, M. y Borjas, B. (2008). La Investigación Acción Participativa: aporte de Fals Borda a la educación popular. Espacio Abierto, 17(4), 615-627. http://www.redalyc.org/articulo.oa?id=12217404\%0ACómo

Ortuño Cano, M.; Gentili, J.; Moretto, B. y Campo, A. (2019). Eventos de exceso hídrico en la prensa escrita (Sistema de Ventania, Argentina). Boletín Geográfico, 1(41), 53-75. https://docs.google.com/viewerng/ viewer?url=http://revele.uncoma.edu.ar/htdoc/revele/index.php/geografia/article/viewFile/2366/59016

Rojas Vilches, O. y Martínez Reyes, C. (2011). Riesgos naturales: evolución y modelos conceptuales. Revista Universitaria de Geografía, 20, 83-116. http://bibliotecadigital.uns.edu.ar/scielo.php?script=sci_arttext\&pi$d=S 1852-42652011001100005 \&$ lng=es\&nrm=iso\&tlng=es

Stein, N. S. (2018). La extensión-comunicación universitaria disputando el lugar de la investigación como fuente de conocimiento. Un recorrido histórico hasta los enfoques actuales. +E: Revista de Extensión Universitaria, 8(9), 14-37. https://doi.org/10.14409/extension.v8i9.jul-dic.7866

UNGRD-IEMP. (2017). Lineamientos para el análisis de la vulnerabilidad social en los estudios de la gestión municipal del riesgo de desastres. http://hdl.handle.net/20.500.11762/24756

United Nations General Assembly (2015). Transforming our world: the 2030 Agenda for Sustainable Development. United Nations General Assembly y United Nations Office for Disaster Risk Redution (2016). Report of the open-ended intergovernmental expert working group on indicators and terminology relating to disaster risk reduction. https://www.preventionweb.net/files/50683_oiewgreportenglish.pdf

Voss, M. y Wagner, K. (2010). Learning from (small) disasters. Natural Hazards, 55(3), 657-669. https://doi. org/10.1007/s11069-010-9498-5 